\title{
Joule heating effect on microdroplet electrowetting platform chip
}

\begin{abstract}
The electrowetting (EW) operation was demonstrated to observe the effect of Joule heating and electrolysis. Joule heating is an electrothermal effect, which is induced from the conductivity of electrode that enables the charge to flow through it. Electrolysis is a decomposition of liquid into gas when it is in direct contact with activated electrode. The experiments were conducted using Potassium Chloride, $\mathrm{KCl}$ solution. The voltage supply used was in $\mathrm{AC}$ and the frequency and voltage were varied. The experiment objective was to see the effect of Joule heating on the flowing of droplet.
\end{abstract}

Keyword: Electrowetting; Joule heating; Microdroplet; Microfluidic 